\title{
STRICT SEPARATION OF CONVEX SETS ${ }^{1}$
}

\author{
V. L. KLEE, JR.
}

A hyperplane in a (real) topological linear space $E$ is a set of the form $f^{-1} c$, where $c$ is a real number and $f$ is a not-identically-zero continuous linear functional on $E$. The hyperplane determines two closed half-spaces $\left.\left.-f^{-1}\right]-\infty, c\right]$ and $f^{-1}[c, \infty[$, and two open halfspaces $\left.-f^{-1}\right]-\infty, c\left[\right.$ and $\left.f^{-1}\right] c, \infty$. Subsets $A$ and $B$ of $E$ are said to be separated $\langle$ strictly separated $\rangle$ by the hyperplane provided $A$ lies in one of the closed 〈open〉 half-spaces and $B$ in the other. An account of the principal known results on separation and strict separation of convex sets may be found in [1] and [3]. Our purpose here is to set forth some apparently new results on strict separation, including the following: If $A$ and $B$ are disjoint closed convex subsets of Euclidean $n$-space and neither contains a ray in its boundary, then $A$ and $B$ can be strictly separated by a hyperplane. A related problem was considered several years ago by the author [3, p. 459], but the present investigation was prompted by a question of Mr. Isaac Namioka.

The proof of our principal result (4) is based on several lemmas, of which the first is given below. A discussion of net-convergence (used below) is contained in [2]. The origin of the linear space under consideration will always be denoted by $\phi$.

(1) Suppose $C$ is a locally compact closed convex subset of a topological linear space, $\phi \in C, U$ is a neighborhood of $\phi$, and $S$ is a net in $C \backslash U$. Then there is a subnet $(x,>)$ of $S$ and a corresponding net $(t,>)$ of positive numbers such that $t_{n}$ converges to a number $t_{0}<\infty$ and $t_{n} x_{n}$ converges to a point $y_{0}$ of $C \backslash\{\phi\}$.

Proof. Since $C$ is closed and locally compact, $U$ contains a neighborhood $V$ of $\phi$ such that the intersection $B$ of $C$ with the boundary of $V$ is compact. For each member $k$ of the domain of $S$ we have $a_{k} S_{k} \in B$ for some $\left.a_{k} \in\right] 0,1[$. The desired conclusion then follows from the fact that $[0,1]$ and $B$ are compact, and $\phi \notin B$.

A subset of a linear space is linearly bounded provided its intersection with each line is bounded. For convex sets, this is equivalent to saying that the set contains no ray.

Presented to the Society, June 18, 1955; received by the editors February 21, 1955.

1 Sponsored by the Office of Ordnance Research, U. S. Army, under Contract DA-04-200-ORD-292. 
(2) With $C$ as in (1), $C$ is compact if and only if $C$ is linearly bounded.

Proof. The "only if" part is trivial, and we wish to show that if $C$ is not compact, then $C$ contains a ray. Now if $C$ is not compact, there exist $S$ and $U$ as in (1) such that $S$ has no convergent subnet. And if $t_{0} \neq 0, x_{n}$ converges to $t_{0}^{-1} y_{0}$, so it follows that $t_{0}=0$. Let $y_{n}=t_{n} x_{n}$ for each $n$; then $\left[0, t_{n}^{-1}\right] y_{n} \subset C$, whence it follows that $\left[0, \infty\left[y_{0} \subset C\right.\right.$, completing the proof of (2).

(3) Suppose $A$ and $B$ are disjoint closed convex subsets of a topological linear space, and there exists a hyperplane $H$ separating $A$ from $B$ such that $A \cap H$ is nonempty and linearly bounded. Then if either $A$ or $B$ is locally compact, there is an open $V \ni \phi$ such that $A+V$ misses $B$.

Proof. Let the continuous linear functional $f$ and the real number $a$ be such that $H=f^{-1} a$ and $\left.\left.A \subset f^{-1}\right]-\infty, a\right]$. Consider the case in which $A$ is locally compact. We wish first to show that the set $C=A \cap f^{-1}[a-1, a]$ is compact, and see by (1) that it suffices to show that $C$ is linearly bounded. Suppose $C$ contains a ray $T$ with initial point $x$, and let $y \in A \cap H$. From the fact that $A$ is closed and convex it follows readily that $A$ contains the ray $T^{\prime}=y+(T-x)$. And since $T \subset f^{-1}[a-1, a]$, it is clear that $f$ is constant on $T$, whence $T^{\prime} \subset A \cap H$, a contradiction showing that $C$ is compact. Now since $C$ is compact and disjoint from the closed set $B$, there exists open $U \ni \phi$ such that $C+U$ misses $B$. Let $\left.V=U \cap f^{-1}\right]-1 / 2,1 / 2$ [. Then $V$ is open, $\phi \in V$, $\left.f^{-1}\right]-\infty, a-1[+V$ misses $B$, and $C+V$ misses $B$, so $V$ is the desired open set.

Consider now the case in which $B$ is locally compact. We may assume $\phi \in B$. Suppose the desired $V$ does not exist, and let $W$ be the set of all open $W \ni \phi$. Then there exists $U \in W$ such that for each $W \in W$ there are points $b_{w} \in B \backslash U$ and $a_{w} \in A$ with $b_{w} \in a_{w}+W$. Now let us apply (1), with the roles of $C$ and $S$ taken by $B$ and $\left(b_{w} \mid W \in W\right.$, $C)$ respectively, to obtain nets $(x,>)$ and $(t,>)$ as described in (1), and let $(u,>)$ be the corresponding subnet of $\left(a_{w} \mid W \in W, C\right)$. If $t_{0}>0$, then $x_{n}$ converges to $t_{0}^{-1} y_{0}$, whence $u_{n}$ converges to $t_{0}^{-1} y_{0}$ and $A \cap B \neq \Lambda$, contradicting one of our hypotheses. Thus $t_{0}=0$, and as in (2), $\left[0, \infty\left[y_{0} \subset B\right.\right.$. Let $p \in A \cap H$ and for each $n$ let $z_{n}=t_{n}\left(u_{n}-p\right)$. Then $z_{n}$ converges to $y_{0}$ and always $p+\left[0, t_{n}^{-1}\right] z_{n} \subset A$, whence $p+\left[0, \infty\left[y_{0} \subset A\right.\right.$. Since the parallel rays $\left[0, \infty\left[y_{0}\right.\right.$ and $p+\left[0, \infty\left[y_{0}\right.\right.$ are separated by the hyperplane $H=f^{-1} a, f$ must be constant on each ray and it follows that $p+\left[0, \infty\left[y_{0} \subset A \cap H\right.\right.$, a contradiction completing the proof of (3).

It is now easy to prove our principal result, which is 
(4) For a locally compact closed convex subset $A$ of a locally convex topological linear space $E$, the following two assertions are equivalent: (i) Whenever $H$ is a hyperplane in $E$ which supports $A$, then $A \cap H$ is linearly bounded. (ii) Whenever $B$ is a closed convex subset of $E \backslash A$ which can be separated from $A$, then $B$ can be separated from $A$ by a hyperplane which misses $A$.

Proof. That (i) implies (ii) follows at once from (3), the local convexity of $E$, and the basic separation theorem for convex bodies. That (ii) implies (i) is obvious when $E$ is the plane, and general proof can be based on this case.

From (4) and the known special separation properties of finitedimensional spaces, we have

(5) For a closed convex subset $A$ of $E^{n}$, the following two assertions are equivalent: (i) $A$ contains no ray in its boundary. (ii) Whenever $B$ is a closed convex subset of $E^{n}$ which misses $A$, then $B$ can be separated from $A$ by a hyperplane which misses $A$.

A partial analogue of (4) can be obtained by applying (3) for the case in which $B$ is locally compact. Other corollaries are obtained by observing that if $A$ and $B$ can be separated by two hyperplanes, one missing $A$ and the other missing $B$, then $A$ and $B$ can be strictly separated. (Together with (5), this yields the result stated in the introduction.) Another consequence of (5) is

(6) Suppose $A$ is a closed convex subset of $E^{n}$ and that $A$ contains no ray in its boundary. Then every linear manifold in $E^{n}$ which misses $A$ is contained in a hyperplane which misses $A$.

In conclusion, we remark that there are examples showing that the assumptions of local compactness cannot be avoided.

\section{REFERENCES}

1. N. Bourbaki, Espaces vectoriels topologiques, Actualités Scientifiques et Industrielles, No. 489, Paris, 1953.

2. J. L. Kelley, Convergence in topology, Duke Math. J. vol. 17 (1950) pp. 277-283.

3. V. L. Klee, Jr., Convex sets in linear spaces, Duke Math. J. vol. 18 (1951) pp. 443-466.

UNIVERSITY OF WASHINGTON 